\title{
An Interactive and Digital Puppeteering Interface for new musical expression (IDPI)
}

\author{
Keywords \\ Interactive System; Signal Processing; Performance animation; Generative Music
}

Puppetry is the oldest form of the virtual reality and has a strong tradition as a theatrical art. The aim of this research project is to create digital puppeteering system which translates gestural acts into live and expressive control of virtual 3D models including in real-time 3D sound. I will devise a model of practice that extends our understanding and notion of the digital puppet. It seeks to establish new practical and conceptual relations between the puppet and new technologies in the framework of puppet theatre. The practical aim is to focus on the special spirit of animated 3D models and silhouettes and to contribute to cultural preservation and fixing of the tradition(s) of puppet theatre. This project will explore the potential of puppetry as a musical expressive medium by new media, including the sensor, 3D sound system, digital projection, and 3D simulation. The conceptual aim of the project is to integrate traditional and new forms of puppetry through different interfaces that will advance traditional forms of cultural expressions. This project focuses on analogies and differences between different puppet theatre traditions. A key aspect is the relationship between the Western puppetry and the Eastern puppetry traditions, and the impact of the resulting cross- cultural dialogue in dramatic performances with figures. In seeking to identify the potential effects of digital puppetry, I will obtain a new vocabulary for gestural musical performance and can develop guidelines that can be used for future creative theatrical practice in the field of digital puppetry. The aim of my research project is to design an interactive digital puppetry system which is sensitive to gestural acts of puppeteers and enriches the performances as a musical expressive medium on its own right. Such a system will serve creative possibilities using digitalisations of old forms by puppet restoration and preserving its instructions. Through analyses of European and Japanese traditional puppet theatres, I will achieve a new cross-cultural form of puppetry. Thus, I investigate how acts and music of puppetry can be restored from not only actual traditional puppet theatre, but also archives and documents, then performed and remediated with digital performance technology. Furthermore, my investigation includes in transitioning layers between old and new media - objects of puppet theatre and digital simulation - alternative action and transformation. I believe that the digital re-presentation of traditional puppetry is one of the most efficient and effective ways to impart to later generations and also to revitalise the arts of puppet theatres. An orientation toward new medias will enable me to explore 'tradition' and the puppet as a technological media object. Through my digital practice and an encounter with old, lost, forgotten puppet theatre, I set out to create something new. 